\title{
The use of pseudo inertia in asymptotic modelling of constraints in boundary value problems
}

\author{
L. Henderson and S. Ilanko \\ Department of Engineering \\ School of Science \& Engineering, \\ The University of Waikato, \\ Private Bag 3105, Hamilton, \\ New Zealand. \\ Ilanko@,waikato.ac.nz
}

\begin{abstract}
In recent publications, the validity of using positive and negative inertial penalty parameters and the advantage of this approach over the conventional positive penalty function approach have been established for linear eigenvalue problems. This paper shows how this method may be applied to solve a boundary value problem. A steady state 2-D heat transfer problem is used to demonstrate the method. First, the governing partial differential equation is modified by adding a pseudo inertial term which results in an equation which is mathematically identical to the equation governing the free vibration of a membrane. The essential boundary conditions of zero temperature along a specified line are imposed using inertial penalty parameters. The characteristic vibration modes found in this way are used to generate the complementary function to the heat transfer problem. This solution satisfies all natural boundary conditions (adiabatic) and zero temperature conditions using the inertial penalty parameter. To satisfy any additional temperature distribution imposed on the system, two sets of corrector terms are superimposed resulting in the final solution. The results are compared with constrained solutions obtained using the Lagrangian multiplier method and the ordinary penalty method.
\end{abstract}

Key Words: pseudo inertia; penalty method; asymptotic modelling; constraints

\section{Introduction}

The penalty method, also known as the artificial spring method in vibration research, is a widely used approach for applying constraints [1-9]. As the accuracy of the solution depends on the magnitude of the penalty parameter, it has been a common practice to select a suitable magnitude by conducting a convergence study [10]. The difficulty in determining an appropriate magnitude for a penalty parameter is that it needs to be sufficiently large to effect a constraint, however, small enough to avoid numerical problems [11-13]. This has been the subject of several recent publications [14-22]. It has been shown that for linear boundary value problems, the use of positive and negative penalty parameters enables the determination of any error due to constraint violation and that it is possible to obtain good approximations to the constrained solution using interpolation of the penalised solutions with respect to inverse penalty parameters. In using negative penalty parameters, one limitation is that the magnitude of the penalty parameter must be larger than the highest critical penalty parameter. For vibration problems this limitation has also been overcome by using positive and negative inertial type penalty parameters instead of the ordinary stiffness type penalty parameters $[17,21]$. This was recently extended to include any linear eigenvalue problem through the use of a generic eigenpenalty parameter [22]. The possibility of using the inertial 
penalty functions in static stress analysis by introducing a virtual inertia was also explored by Ilanko and Kreuzer [23]. In this work, the stiffness of a structure was determined by finding the natural frequency of an equivalent vibratory system. Although a distribution of mass is not needed for static analysis, in order to determine the natural frequencies, a virtual inertia was introduced to create a vibratory system and a large artificial mass was applied to suppress the motion corresponding to a constraint. The terms virtual and artificial inertia are used to indicate the difference in the purpose of the introduced mass. Virtual inertia is used to obtain the vibration mode(s) of an otherwise non-vibratory system while the artificial inertia is used to enforce a constraint. Using a large value for the artificial mass helps to prevent its movement effectively constraining the corresponding degree of freedom, and vibration analysis of the system with the vibrating virtual mass gives the required mode. The method proved successful but when attempting to apply this approach to solve a heat transfer problem after introducing a distribution of pseudo mass (unlike a structural system where an actual mass or inertia makes it a vibratory system, for a heat transfer problem since the term mass has no physical meaning, instead of virtual mass we use the term pseudo mass), effectively creating a membrane vibration problem, the following challenges emerged.

It was found that while eigenpenalty functions can be successfully used to effect homogeneous Dirichlet boundary conditions (constraints such as zero temperature in a heat transfer problem or zero displacement in a vibration problem), imposition of inhomogeneous Dirichlet conditions (prescribing temperature distribution along a boundary or imposing a displacement profile in a structure) was not straightforward. The reason for this is the fact that the vibratory characteristics of a linear system depends only on the constraints during vibration and not on any existing static displacements. Thus the non-homogeneous Dirichlet type boundary conditions cannot be satisfied merely by superposition of the natural modes. This meant the inhomogeneous conditions had to be met by adding corrector terms which only changed the boundary values without affecting the governing equations throughout the domain of interest. However, the addition of a suitable temperature distribution throughout the plate domain to match the prescribed distribution at a boundary caused a violation of the governing equation effectively resulting in an additional distribution of heat input. Application of an equal and opposite heat input to correct this resulted in violation of the adiabatic conditions (but the conditions at the boundary subject to an imposed temperature distribution remained unchanged due to the presence of the penalty terms). Therefore a final correction had to be made by applying heat input at the insulated boundaries. Thus the procedure consisted of four major steps. These were as follows:

1.) First a set of orthogonal functions that satisfied adiabatic conditions along some edges, and zero temperature along an edge which was to be subject to a prescribed temperature distribution was generated.

2.) In order to correct the temperature distribution at the boundary where the non-zero prescribed distribution was to be imposed, an extra temperature distribution was introduced over the full domain in the form of a low order polynomial. This had the effect of an imposed heat input distribution. This was removed by applying an equal and opposite heat distribution. Violations to the adiabatic boundary conditions were additionally corrected by applying an equal and opposite heat flow distribution along the relevant edges.

3.) The overall PDE was solved using the characteristic functions generated in step 1, taking into account the corrector heat generation terms obtained in step 2. 
4.) Finally the low order polynomial chosen as part of step 2 was superimposed to produce the final solution.

It should be noted here that once the orthogonal characteristic temperature modes were determined, solving the PDEs meant summing the coefficients of a series only and did not require a significant effort.

It is useful to compare the procedure we propose with an enhanced version of the method of eigenfunction expansions (MME) presented by Shankar [24] in which solutions to boundary value problems were generated using eigenfunctions. In [24] eigenfunctions corresponding to a larger embedding domain were used to generate the solution to a given boundary value problem, thus removing the limitation of the type of geometries that could be solved using the MME. In the present work, eigenfunctions are generated for the actual domain but the boundary conditions are satisfied by using the procedure described above.

\section{Theoretical Derivations}

(a) Solving a 2-D steady-state heat transfer problem with prescribed temperature distribution along one edge using the vibration modes of an equivalent membrane

The governing partial differential equation for steady-state two dimensional heat transfer in a rectangular plate with constant thermal conductivity is

$$
\frac{\partial^{2} T}{\partial x^{2}}+\frac{\partial^{2} T}{\partial y^{2}}+\frac{\dot{q}_{G}}{k_{\text {cond }}}=0
$$

where $\dot{q}_{G}$ represents the net heat generation in the plate and $k_{\text {cond }}$ is the conductivity vector. Using Galerkin's method, for a suitable choice of a series approximation for the temperature $T$, this may be discretised to obtain a matrix equation of the form

$$
[D]\{C\}=\{H\}
$$

Here $D$ is the derived conducivity matrix, $H$ is the heat generation vector, [19] and $C$ is the undetermined weighting coefficients. It is useful to point out at this stage that equations (2.1) and (2.2) are analagous to those used in solving a two-dimensional membrane deflection problem; where conductivity matrix, $D$, is analogous to the membrane stiffness matrix and $H$ is analogous to an applied pressure at the membrane's surface. The field variable $T$ which represents the temperature distribution in a heat transfer problem would represent the deflection in the membrane problem.

We will consider a general two dimensional heat transfer case where a prescribed temperature distribution, $\zeta(y)$ is applied along one of the edges and adiabatic boundary conditions are imposed along the remaining three.

Before solving Eq. (2.2), a pseudo vibration problem is constructed that will produce mode shapes with zero values of the field variable at the edge where the temperature distribution is to be applied. These mode shapes will later be used as admissible functions in Eq. (2.1). Mode shapes are assumed to be the form

$$
T_{r}(x, y)=\sum_{i=1}^{n} \sum_{j=1}^{n} \hat{C}_{r} f_{i}(x) g_{j}(y) \quad \text { where } r=j+(i-1) n,
$$


where functions $f$ and $g$ are continuous over the domain of the plate. The corresponding eigenvalue problem is of the form

$$
[D]\{\hat{C}\}=\lambda[M]\{\hat{C}\}
$$

Eq. (2.4) is now analogous to a membrane vibration problem, where the conductivity matrix, $D$, is identical to that used in equation Eq. (2.2). The $M$ matrix is derived assuming a uniform pseudo mass density over the area of the plate. For convenience this has been taken as unity. We additionally include a penalty mass term in the $M$ matrix entries such that a distributed penalty mass of $\gamma$ magnitude is applied along one of the four edges. As has already been demonstrated by Ilanko and Tucker [19]. the conductivity matrix $D$ may be given by

$$
D_{r, s}=k_{\text {cond }}\left(\int_{x=0}^{a} f_{i}^{\prime} f_{k}^{\prime} d x \int_{y=0}^{b} g_{j} g_{l} d y+\int_{x=0}^{a} f_{i} f_{k} d x \int_{y=0}^{b} g_{j}^{\prime} g_{l}^{\prime} d y\right) \text { where } s=l+(k-1) n
$$

The pseudo mass matrix for a unifrom plate may be expressed as

$$
M_{r, s}=\int_{x=0}^{a} f_{i} f_{k} d x \int_{y=0}^{b} g_{j} g_{l} d y+\gamma\left(f_{i}(a) f_{k}(a) \int_{y=0}^{b} g_{j} g_{l} d y\right)
$$

Eq. (2.4) can now be solved to produce the desired mode shapes, each with zero temperature at the required edge, $(x=a$ in this case $)$.

In order to bring the temperature at the edge $x=a$ up to the required temperature distribution, $\zeta(y)$, a suitable additive function must be selected which can be superimposed onto the final solution. An obvious choice is simply $\zeta(y)$. However the effects of adding such a function must be taken into consideration such that the overall partial differential equation can still be satisfied. A suitable 'heat generation' corrector term can be generated by applying Eq. (2.1) to $\zeta(y)$, as shown below

$$
k_{\text {cond }}\left[\frac{\partial^{2}(\zeta(y))}{\partial x^{2}}+\frac{\partial^{2}(\zeta(y))}{\partial y^{2}}\right]=\dot{q}_{c o r r 1}
$$

In addition to this, a second 'heat flow' corrector term must be created to ensure that any required adiabatic conditions are satisfied. At such edges the first partial derivative in the normal direction must be zero. Such corrector terms are required along edges $y=0$ and $y=b$ for the case discussed. These are equated as follows

$$
\begin{aligned}
& -\left.k_{\text {cond }} \frac{\partial(\zeta(y))}{\partial y}\right|_{y=o}=q_{c o r r 2} \\
& -\left.k_{\text {cond }} \frac{\partial(\zeta(y))}{\partial y}\right|_{y=b}=q_{c o r r 3}
\end{aligned}
$$

Eq. (2.2) can now be solved using the $D$ matrix defined in Eq. (2.5) and the heat generation vector, $H$, shown below, where once again $r=j+(i-1) n$. 


$$
H_{r}=\sum_{i=1}^{n} \sum_{j=1}^{n} \frac{\hat{C}_{r, s}}{k_{\text {cond }}} \int_{0}^{b} \int_{0}^{b}\left[\dot{q}_{c o r r 1} f_{i}(x) g_{j}(y)+q_{c o r r 2} f_{i}(x) g_{j}(0)+q_{c o r r 3} f_{i}(x) g_{j}(b)\right] \cdot d x d y
$$

It is worth noting that since the same D matrix was used in the solution of the eigenvalue equation (2.4) computational procedures such as LU decomposition need not be repeated. Once the $C$ vector has been obtained, the final steady-state temperature distribution of the plate can be expressed in the form

$$
T(x, y)=\sum_{s=1}^{n n} C_{s} \sum_{r=1}^{n n} \hat{C}_{r, s} f_{i}(x) g_{j}(y)+\zeta(y)
$$

\section{(b) Alternative solutions}

Lagrangian multipliers provide a relatively simple means of imposing constraints in a variational problem. This method however requires line constraints to be approximated as a series of points, each point requiring an additional row and column in the overall system matrix. The additional equations required to impose each prescribed point are of the form

$$
\sum_{i=1}^{n} \sum_{j=1}^{n} A_{r} f_{i}\left(x_{u}\right) g_{j}\left(y_{u}\right)-T_{u}=0
$$

An additional energy term must also be included into Galerkin's formulation for each constraint added. This is shown below

$$
\begin{aligned}
& \int_{x=0}^{a} \int_{y=0}^{b} f_{k}(x) g_{l}(y)\left(\sum_{i=1}^{n} \sum_{j=1}^{n} A_{r}\left(f_{i}^{\prime \prime}(x) g_{j}(y)+f_{i}(x) g_{j}^{\prime \prime}(y)\right)\right) d x d y+ \\
& \frac{\partial}{\partial A_{r}}\left(A_{m n+1}\left(\sum_{i=1}^{n} \sum_{j=1}^{n} A_{r} f_{i}\left(x_{1}\right) g_{j}\left(y_{1}\right)-T_{1}\right)+A_{m n+2}\left(\sum_{i=1}^{n} \sum_{j=1}^{n} A_{r} f_{i}\left(x_{2}\right) g_{j}\left(y_{2}\right)-T_{2}\right)+\ldots\right)=0,
\end{aligned}
$$

where $A_{m n+u}$ is the Lagrangian multiplier corresponding to the $u^{\text {th }}$ constraint. Unlike the pseudo vibration technique described in $(a)$, the Lagrangian method allows the steady-state temperature distribution to be determined upon solving a single set of simultaneous equations. The overall matrix equation is of the form

$$
[\hat{D}]\{A\}=\{H\}
$$

The $\hat{D}$ entries are identical to those described in Eq. (2.5) for $r$ and $s$ terms less than $n^{2}$, rearranging (2.12) produces the additional $\hat{D}$ required terms as follows

$$
\hat{D}_{n n+u, s}=\hat{D}_{r, n n+u}=f_{i}\left(x_{u}\right) g_{j}\left(y_{u}\right)
$$

Additional terms are also required in the $A$ and $H$ vectors, these are

$$
\begin{aligned}
& A_{n n+u}=\lambda_{u} \\
& H_{n n+u}=T_{u}
\end{aligned}
$$


Solving Eq. (2.13) gives a solution of the form

$$
T(x, y)=\sum_{i=1}^{n} \sum_{j=1}^{n} A_{r} f_{i}(x) g_{j}(y)
$$

Another alternative is the conventional penalty parameter technique, which also allows a solution to be obtained without solving an eigenvalue problem. Once again the governing matrix equation is of the form shown in Eq. (2.13). However, in this case, a penalty is imposed on any deviation from the prescribed temperature distribution, $\zeta(y)$, at the edge $x=$ $a$. This penalty is incorporated into the overall conductivity and heat generation terms as a potential energy term. This is analogous to the potential energy stored in a spring of large stiffness in a membrane deflection problem and is given as follows,

$$
\frac{\alpha}{2} \int_{0}^{b}\left(\sum_{i=1}^{n} \sum_{j=1}^{n} A_{r} f_{i}(a) g_{j}(y)-\zeta(y)\right)^{2} d y
$$

where $\alpha$ is a penalty parameter of sufficiently large magnitude. Unlike the Lagrangian multiplier technique, the penalty parameter technique does not require additional rows or columns in the system matrices. Eq. (2.17) is simply included in the overall partial differential equation, resulting in an additional term which must be included in the conductivity entries equated in Eq. (2.5). This is shown below

$$
D_{p e n}=\alpha \int_{0}^{b} f_{i}(1) f_{k}(1) g_{j}(y) g_{l}(y) d y
$$

Incorporating Eq. (2.18) into the overall partial differential equation also produces a heat generation vector with entries of the form

$$
H_{s}=\alpha \int_{0}^{b} f_{k}(1) g_{l}(y) \zeta(y) d y
$$

The penalty parameter technique once again produces a solution of the form shown in Eq. (2.17).

\section{Results and Discussion}

As an illustrative example, a simple two dimenional steady-state heat transfer problem will be considered with a prescribed parabolic temperature distribution along the edge $x=a$. $\zeta(y)$ will arbitrarily be taken as

$$
\zeta(y)=4 \hat{T} \frac{y}{b}\left(1-\frac{y}{b}\right) \text { where } \hat{T}=100^{\circ} \mathrm{C}
$$

The reamaining three sides will be taken as adiabatic. Plate dimensions will be taken as unity for ease of calculation. Polynomial shape functions of the following form will be used 


$$
\begin{aligned}
f_{i}(x) & =\left(\frac{x}{a}\right)^{i-1} \\
g_{j}(y) & =\left(\frac{y}{b}\right)^{j-1}
\end{aligned}
$$

This is the same problem considered by Ilanko and Tucker [19], in which the use of positive and negative values for the conventional penalty parameter was advocated. This example provides a relatively difficult case to model as the prescribed boundary conditions create singularities at two of the corners. At points $(1,0)$ and $(1,1)$ the slope imposed by $\zeta(y)$ directly conflicts with the adiabatic conditions along edges $y=0$ and $y=1$. Analytical solutions are readily available for this situation [25], and will be used to assess the accuracy of the results obtained using the inertial penalty technique.

Combining equations (3.2a) and (3.2b) with equations (2.5) and (2.6) gives the conductivity and pseudo mass matrices for this case. Carrying out the required integration gives

$$
\begin{gathered}
D_{r, s}=k_{\text {cond }} \frac{j l b^{(j+l-3)} a^{(i+k-1)}}{(j+l-3) \cdot(i+k-1)} \\
M_{r, s}=\frac{a^{(i+k-1)} b^{(j+l-1)}}{(i+k-1) \cdot(j+l-1)}+\gamma \frac{a^{(i+k-2)} b^{(j+l-1)}}{(j+l-1)}
\end{gathered}
$$

Equating equations (2.7), (2.8a) and (2.8b) and combining with Eq. (2.9) gives the heat generation vector

$$
H_{s}=\sum_{i=1}^{n} \sum_{j=1}^{n} 8 \hat{T} \hat{C}_{r, s}\left[\frac{a^{i} b^{j}}{i j}-\beta\right]
$$

where $\beta=\frac{a}{b i}$ for $j=0$, and $\beta=0$ for $j>1$

Equations (2.2) and (2.4) can now be solved using matrix algebra. Figure 1 shows the steady-state temperature distribution predicted using $n=7$, and $\gamma=50$.

As demonstrated by Carslaw and Jaeger [25], standard procedures yield an analytical solution for this problem of the form

$$
T=\frac{2}{3} \hat{T}-\frac{4 \hat{T}}{\pi^{2}} \sum_{i=2}^{\infty} \frac{\cosh \left((2 i-2) \pi\left(\frac{x}{b}+\frac{a}{2 b}\right)\right)}{(i-1)^{2} \cosh \left((2 i-2) \pi \frac{a}{b}\right)} \cos \left((2 i-2) \pi\left(\frac{y}{b}+\frac{1}{2}\right)\right)
$$

The inertial penalty solution, (shown in figure 1) compares well with the analytical solution which has been evaluated in a recent publication [19]; it also behaves correctly about the two singularities, (as mentioned earlier these occur at $(1,0)$ and $(1,1)$ ). Some deviation can however be seen along the edges $y=0$ and $y=1$. This deviation was also seen in the Lagrangian multiplier solution obtained using $n=7$ and is most likely due to the relatively low number of terms being used in both cases, (the analytical solution shown in Figure 2 was constructed using 1500 shapes whereas penalty inertial solution was obtained using only 49 
$(n \times n))$. In terms of computational power both the Lagrangian multiplier method and the penalty inertia technique have slight advantages over the analytical solution. The predictions obtained using 49 terms for both penalty techniques were slightly more accurate than those obtained when the analytical solution is truncated to the same size. The Lagrangian multiplier method allowed more terms to be used than the inertial penalty technique before numerical instabilities arose and, for this example, modelled the prescribed temperature distribution extremely well.

The results obtained using the inertial penalty method are compared to those obtained using both the ordinary penalty method and the Lagrangian multiplier method in Table 1 . To ensure a fair comparison, $n$ was taken as 5 in all three cases; this allowed a range of penalty magnitudes to be trialled without introducing numerical overflow in the inertial penalty solutions. As has already been discussed, solutions were obtained with the inertial penalty method using up to 7 terms, however with $n=7$ the magnitude of $\gamma$ was limited to 50 and less. The same polynomial shape functions were used in each case. Eleven equally spaced points were used to approximate $\zeta(y)$ in the Lagrangian multiplier solution.

Table 1 shows that both the inertial penalty, and ordinary penalty methods produce results which converge towards the equivalent Lagrangian multiplier solution. Table 1 also suggests that the inertial penalty parameters of only one-tenth the magnitude are required to obtain results similar to those produced using the conventional penalty parameter technique.

Although increases in magnitude of the inertial penalty parameter were observed to produce more accurate results, upper limits were seen above which results no longer converged due to large round-off errors. For $n=5$, inertial penalty magnitudes greater than 1000 were found to produce complex mode shapes and inaccurate temperature predictions. As the number of mode shapes used was increased the upper limit was found to decrease; when $n$ was increased to 7 the maximum magnitude was reduced to 50 and with $n=8$ the maximum was only 12 . Similar problems were also observed when using the ordinary penalty parameter method. For this problem a maximum penalty parameter magnitude of $10^{5}$ was observed for $n=5$. A more effective way of obtaining accurate solutions is shown in the second column of table 1 . Instead of using increasingly large penalty values a combination of positive and negative penalty values is used to predict the constrained solution as recommended in two recent publications $[20,21]$.

Figure 2 illustrates the convergence and bounding nature of the results obtained using the inertial penalty method. It also shows how positive and negative inertially penalised solutions approach the corresponding Lagrangian solution from opposite sides. This bracketing effect has obvious benefits as it allows the maximum tolerance in any prediction to be determined, (as shown by the $\left|T_{+\gamma}-T_{-\gamma}\right| / 2$ and $\left|T_{+\alpha}-T_{-\alpha}\right| / 2$ columns in Table 1).

One interesting observation which can be made regarding Table 1 and Figure 2 is that, for $n=$ 5 , neither the inertial penalty method nor the ordinary penalty parameter method bracket the analytical solution calculated using equation (3.6). Table 2 however shows that increasing the number of terms used in the series produces results which converge to the analytical value.

It may be noted that $n=7$ produces results which bracket the analytical solution within $\pm 0.0001 \mathrm{~K}$. Results could not be obtained for $n$ values greater than 8 , even when low magnitude inertial penalty parameters were used. At higher $n$ values numerical overflow problems were once again encountered. These issues were most likely due to the increasing 
order of the polynomial shape functions used. It may be possible to overcome this problem by selecting alternative shape functions, which are less likely to produce excessively large or small numbers.

The results presented in this paper show that, pseudo inertial terms can be used to solve a steady state heat transfer problem. However, in relation to the conventional penalty parameter method, the authors found the technique to be somewhat laborious. Nevertheless, there is a slight advantage in using the inertial penalty method. Unlike the ordinary penalty parameter method, the inertial penalty technique does not exhibit any critical penalty values, $[16,19]$. In the ordinary penalty parameter technique, if the magnitude of the negative penalty parameter used is less than the critical penalty values, the results may be unreliable. It is also possible to obtain bracketing solutions to the constrained problem by using a combination of positive ordinary penalty terms and positive inertial penalty terms. Additionally it must be noted that mode shapes need only be generated once and can be used to solve a range of different problems without the need to repeatedly solve the eigenvalue problem. The orthogonality conditions used enable relatively fast computation times once the mode shapes have been generated.

\section{Concluding Remarks}

A two dimensional steady state heat transfer problem involving adiabatic conditions and prescribed temperature distributions along boundaries has been solved using a penalty method. A pseudo inertia distribution was introduced to the system to obtain characteristic orthogonal functions that satisfy zero temperature along boundaries which are to be subject to a prescribed temperature distribution. The zero temperature distribution was effected by adding large inertial type penalty terms. Further temperature distributions were imposed on the system in the form of low order polynomials to satisfy the boundary conditions. The new heat flow distribution generated by the added temperature distribution was cancelled by the application of an equal and opposite distribution for which the resulting temperature profile was determined. The final temperature distribution found by superposition agreed well with results generated from alternative methods. The results show that the solution obtained in this way converges towards the constrained solution as the magnitude of the inertial penalty parameter increases irrespective of the sign of the penalty term. Furthermore, the constrained solution was found to be bounded by results obtained using positive and negative inertial penalty terms.

This shows that the inertial penalty method can be applied to generate the characteristic orthogonal modes of a system defined by boundary value problems if a distribution of pseudo inertia and inertial penalty terms are introduced. Depending on the nature of the boundary value problems, several steps are needed to obtain the final solution. While this appears to be a disadvantage, it should be noted here that once the orthogonal functions are found, all remaining calculations require only basic matrix algebra and the solution is generated by computing the terms in a series. 


\section{References}

1. Amabili M. 1997, Shell-plate interaction in the free vibrations of circular cylindrical tanks partially filled with a liquid : The artificial spring method Journal of Sound and Vibration 199: 431-452.

2. Amabili M, Garziera R. 1999, A technique for the systematic choice of admissible functions in the Rayleigh-Ritz method. Journal of Sound and Vibration 224: 519-539.

3. Gavete L, et al. 2000 Implementation of essential boundary conditions in a meshless method. Communications in Numerical Methods in Engineering 16: 409-421.

4. Pannachet $\mathrm{T} \&$ Askes H. 2000 Some observations on the enforcement of constraint equations in the EFG method. Communications in Numerical Methods in Engineering 16: 819-830.

5. Yuan, J \& Dickinson, S.M. 1992, On the use of artificial springs in the study of the free vibrations of systems comprised of straight and curved beams. Journal of Sound and Vibration 153: 203-216.

6. Yuan, J \& Dickinson, S.M. 1992, The flexural vibration of rectangular plate systems approached by using artificial springs in the Rayleigh-Ritz method. Journal of Sound and Vibration 159: 3955.

7. Cheng, L, \& Richard M.J. 1995 A new formulation for the vibration analysis of a cylindrical vessel containing fluid via the use of artificial spring systems. Thin-walled Structures 21: 17-30.

8. Tomioka T et al. 1996 Analysis of free vibration of rotating disk-blade coupled systems by using artificial springs and orthogonal polynomials. Journal of Sound and Vibration 191: 53-73.

9. Ezawa Y. \& Okamoto N. 1996 Development of contact stress analysis programs using the hybrid method of FEM and BEM. Computers and Structures 58: 13-20

10. Singh I. V \& Tanaka M, 2006 Heat transfer analysis of composite slabs using meshless elementfree Galerkin method, Computational Mechanics 38, pp 521-532

11. Courant R. 1943 Variational methods for the solution of problems of equilibrium and vibration. Bulletin of the American Mathematical Society 49: 1-23.

12. Zienkiewicz OC. 1974 Constrained Variational Principles and Penalty Function Methods in the Finite Element Analysis. Lecture Notes in Mathematics. Springer: Berlin, 363; 207-214.

13. Zienkiewicz OC. 1977 The Finite Element Method, McGraw-Hill: London; Third Edition; 86; 284-287.

14. Ilanko S, \& Dickinson SM. 1999, Asymptotic modelling of rigid boundaries and connections in the Rayleigh-Ritz Method. Journal of Sound and Vibration 219: 370-378.

15. Ilanko, S. 2002 Existence of natural frequencies of systems with imaginary restraints and their convergence in asymptotic modelling, Journal of Sound and Vibration 255 (5), 883-898

16. Ilanko, S. 2002 The use of negative penalty functions in constrained variational problems, Communications in Numerical Methods in Engineering 18: 659-668 
17. Ilanko, S. 2005 Introducing the use of positive and negative inertial functions in asymptotic modelling, Proc. R. Soc. A 461, (2060), 2005: 2545-2562.

18. Ilanko S. 2005 Asymptotic modelling theorems for the static analysis of linear elastic structures. Proc. R. Soc. A 461 (2063): 3525-3542.

19. Ilanko, S. \& Tucker. A.S. 2005 The use of negative penalty functions in solving partial differential equations, Communications in Numerical Methods in Engineering, 21, 99-106.

20. Askes, H., \& Ilanko, S. 2006 The use of negative penalty functions in linear systems of equations, Proc. R. Soc. A 462 (2074): 2965 - 2975

21. Williams F.W \& Ilanko S. 2006 The use of the reciprocals of positive and negative inertial functions in asymptotic modelling. Proc. R. Soc. A, 462 (2070):1909-1915.

22. Ilanko, S. \& Williams, F.W. 2008 Wittrick-Williams algorithm proof of bracketing and convergence theorems for eigenvalues of constrained structures with positive and negative penalty parameters, International Journal for Numerical Methods in Engineering, 75 (1), pp 83 102

23. Ilanko. S. \& Kreuzer, E. 2005, Virtual Inertia and Penalty Functions for Variational Problems, (In, Advances in applied mechanics. Edited by Xie, Y.M., Mouritz, A.P., Afaghi Khatibi, A., Gardiner, C. and Chiu, W.K. Published by Institute of materials engineering, Australia Ld., Melbourne 2005) - presented at the Fourth Australasian Congress of Applied Mechanics, February pp 137-144.

24. Shankar, P.N., 2005, Eigenfunction expansions on arbitrary domains, Proc. R. Soc. A 461 (2059): $2121-2133$

25. Carslaw H. S \& Jaeger J.C., 1959 Conduction of Heat in Solids, Second Edition, Oxford University Press, Oxford, pp 162-169. 
Table 1: Temperature at the Centre of the Plate using Inertial and Ordinary Penalty Parameter Methods $(n=5)$

\begin{tabular}{|c|c|c|c|c|c|c|}
\hline \multirow{2}{*}{$\begin{array}{c}\text { Penalty } \\
\text { Magnitude }\end{array}$} & \multicolumn{2}{|c|}{ Penalty Inertia Technique } & \multicolumn{2}{|c|}{$\begin{array}{c}\text { Penalty Parameter } \\
\text { Technique }\end{array}$} & \multirow{2}{*}{$T_{\text {Lagrangian }}$} & \multirow{2}{*}{$T_{\text {Analytical }}$} \\
\cline { 2 - 6 } & $\frac{\left(T_{+\gamma}+T_{-\gamma}\right)}{2}$ & $\frac{\left|T_{+\gamma}-T_{-\gamma}\right|}{2}$ & $\frac{\left(T_{+\alpha}+T_{-\alpha}\right)}{2}$ & $\frac{\left|T_{+\alpha}-T_{-\alpha}\right|}{2}$ & & \\
\hline \hline $10^{5}$ & --- & --- & $68.2528 \mathrm{~K}$ & $0.0002 \mathrm{~K}$ & $68.253 \mathrm{~K}$ & $68.402 \mathrm{~K}$ \\
\hline $10^{4}$ & --- & --- & $68.253 \mathrm{~K}$ & $0.0006 \mathrm{~K}$ & $68.253 \mathrm{~K}$ & $68.402 \mathrm{~K}$ \\
\hline $10^{3}$ & $68.253 \mathrm{~K}$ & $0.0005 \mathrm{~K}$ & $68.2757 \mathrm{~K}$ & $0.0288 \mathrm{~K}$ & $68.253 \mathrm{~K}$ & $68.402 \mathrm{~K}$ \\
\hline $10^{2}$ & $68.253 \mathrm{~K}$ & $0.0053 \mathrm{~K}$ & $68.2468 \mathrm{~K}$ & $0.0577 \mathrm{~K}$ & $68.253 \mathrm{~K}$ & $68.402 \mathrm{~K}$ \\
\hline $10^{1}$ & $68.2537 \mathrm{~K}$ & $0.0533 \mathrm{~K}$ & $69.7589 \mathrm{~K}$ & $2.0472 \mathrm{~K}$ & $68.253 \mathrm{~K}$ & $68.402 \mathrm{~K}$ \\
\hline $10^{0}$ & $68.3237 \mathrm{~K}$ & $0.5357 \mathrm{~K}$ & --- & --- & $68.253 \mathrm{~K}$ & $68.402 \mathrm{~K}$ \\
\hline
\end{tabular}


Table 2: Convergence of Inertially Penalised Solution at Plate Centre towards the Analytical Solution, $\quad(\gamma=50)$

\begin{tabular}{|l|l|l|l|}
\hline \multicolumn{1}{|c|}{$n$} & $\frac{\left(T_{+\gamma}+T_{-\gamma}\right)}{2}$ & \multicolumn{1}{|c|}{$T_{\text {Lagrangian }}$} & \multicolumn{1}{|c|}{$T_{\text {Analytical }}$} \\
\hline 3 & $65.2778 \mathrm{~K}$ & --- & $68.402 \mathrm{~K}$ \\
\hline 4 & $65.5261 \mathrm{~K}$ & $65.5260 \mathrm{~K}$ & $68.402 \mathrm{~K}$ \\
\hline 5 & $68.2531 \mathrm{~K}$ & $68.2530 \mathrm{~K}$ & $68.402 \mathrm{~K}$ \\
\hline 6 & $68.2725 \mathrm{~K}$ & $68.2725 \mathrm{~K}$ & $68.402 \mathrm{~K}$ \\
\hline 7 & $68.4028 \mathrm{~K}$ & $68.3796 \mathrm{~K}$ & $68.402 \mathrm{~K}$ \\
\hline
\end{tabular}




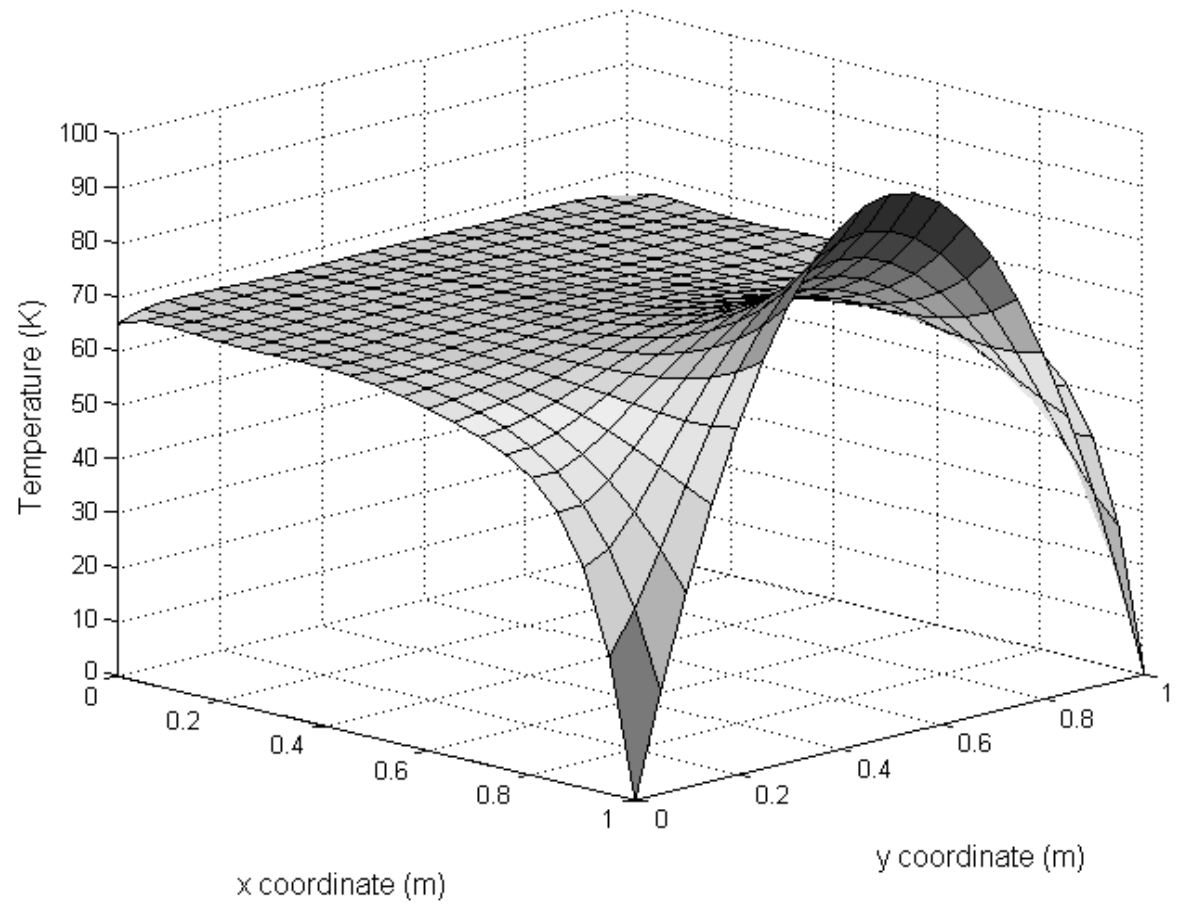

Figure 1: Steady-State Temperature Distribution Predicted Using $n=7$ and $\gamma=50$ 


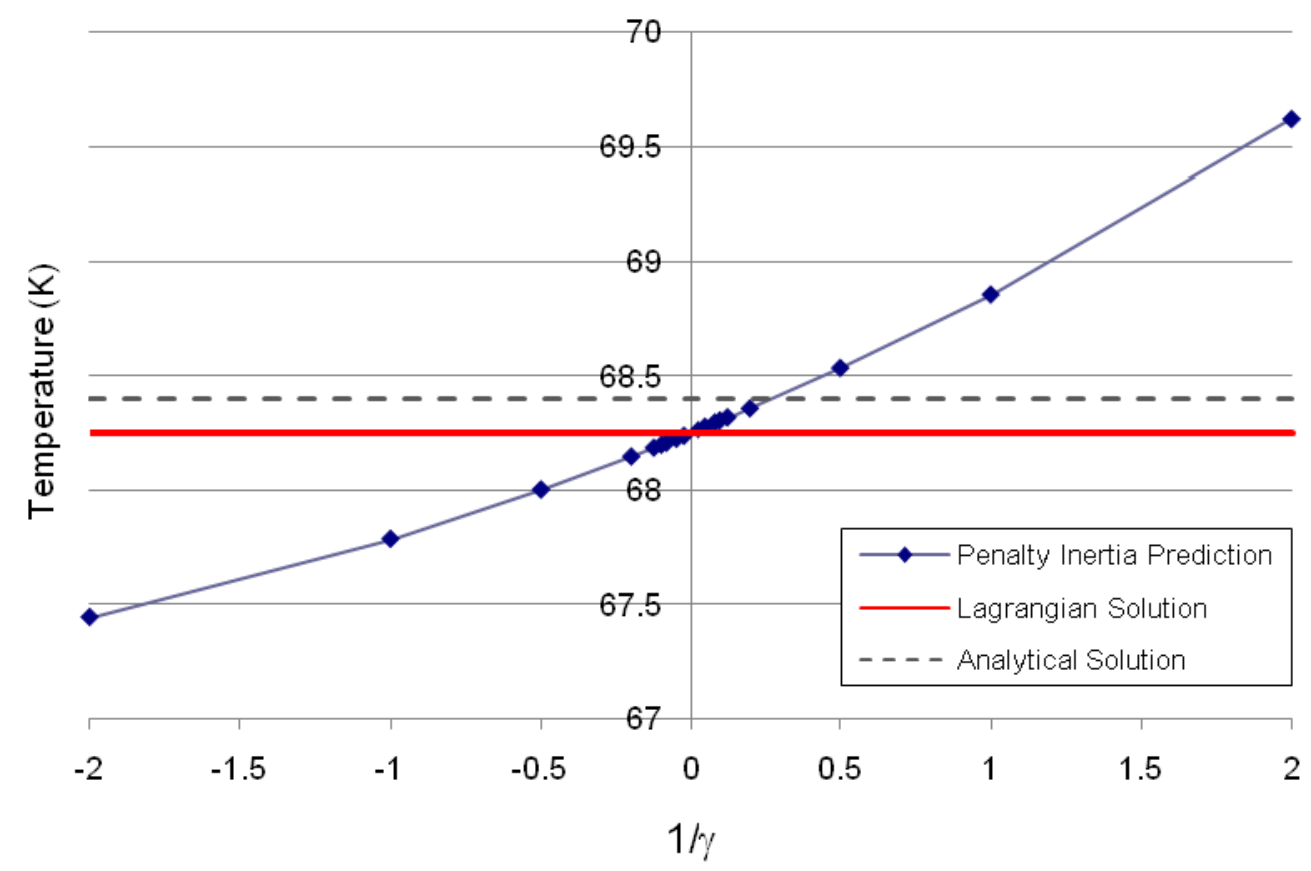

Figure 2: Temperature at Centre of Plate Predicted Using Various Magnitudes of Inertial Penalty Parameter, $(n=5)$ 